Volume 10, No.6, November - December 2021

International Journal of Advanced Trends in Computer Science and Engineering

Available Online at http://www.warse.org/IJATCSE/static/pdf/file/ijatcse091062021.pdf

https://doi.org/10.30534/ijatcse/2021/091062021

\title{
Ontology of Heterogeneous Image File Formats and their Disparate Applications
}

\author{
Kauthar Abdulazeez ${ }^{1}$, Sohit Agarwal ${ }^{2}$ \\ ${ }^{1}$ Suresh Gyan Vihar University, Jaipur, India, kauthar.abdulazeez@ gmail.com \\ ${ }^{2}$ Suresh Gyan Vihar University, Jaipur, India, Sohit.agarwal@mygyanvihar.com
}

Received Date : October 07, 2021 Accepted Date : November 10, 2021 Published Date : December 06, 2021

\begin{abstract}
Different image formats are available in the world today which are used for various purposes, this paper elaborates the Ontology of different Image File Formats and their various applications. Digital images are saved in various Image File Formats which have different properties and features which are ideal for a particular use. A digital image is primarily classified into two types, raster or vector type. Image format elucidate how the information in the image will be stored. Image file format is a systematic way of storing and arranging digital images. Image file format can store data in compressed format (which may be lossy or lossless), uncompressed format or a vector format. Some Image format are suitable for a particular purpose while some are not. TIFF Image type is good for printing whereas PNG or JPG, are best for web. Analysis of the basic Image File Format have been carried out practically and the result is displayed in the coming section.
\end{abstract}

Key words: Digital Image, Image format, compressed, uncompressed, Raster, Vector.

\section{INTRODUCTION}

Imagine the world today without images, images have played a crucial role in this digital era. Digital image is a numerical 2D or 3D illustration of a physical object or scene [30]. Digital images are taken using any device that is facilitated with camera or having ability to capture any visual scene. Images are used for communication in a visual view of the information to be passed. The image can be stored using different measures of image file formats. Digital images are stored in various format. Every image format has its own features associated to it. Today, different image formats are available which are used for various purposes, this paper elaborates the Ontology of different Image File Formats and their varying applications, Digital images are saved in numerous Image File Formats which have different properties and features which are ideal for a particular use. Transferring images from time to time is required for easy access, change of view, x-ray images, documentation and other purposes thus, they can be compressed slightly or more subject to the use.

\section{IMAGE FILE FORMAT}

Image File format continue to evolve, becoming more complex as revised software versions inculcate new features or functionalities. An image file format is a standard specification for organizing and storing digital images, the information in the Image is encoded into bits of data for storage. All images are having a particular extension attributed to it, which identifies the type of format used to store the image. Numerous image file format are used nowadays which have some unique features and these features are associated with the image stored in that image format. Conventional Image file format have a unique three letter extension that follows the name of the image. The extension passes information to the operating system on the type of image format and the type of application that must be used to open it.

An Image encoded into bits of data and stored in a specific image format is recognized as an image, and it provides useful information such as its matrix size to aid communication with the file. Any program which adheres to the standard of the format

can open the file and display the image.

\section{CLASSIFICATION OF IMAGE FILE FORMAT}

Digital Images are classified into Raster and Vector format, they can also be stored based on these formats. Raster image also called bitmap image is a type of image composed of picture elements organized in a grid of pixels to form an image [34]. These pixels depicts a number of bits like color depth, color etc.

Raster images are composed of grid of pixels organized to form an image, therefore resizing it will alter the quality of the image. If the resolution is low, the image should be small to conserve the quality. Raster images are prioritized for painting.

While vector image consist of paths made up of curves, lines, angles and points that works on

geometric representation or mathematical equation. Vector images are smooth at any feasible size. A vector image does not depend on the resolution, hence the output quality is not affected if the image is contracted or expanded. 


\subsection{Difference between Raster and vector Images}

The table below distinguish the differences, advantages, and disadvantages between raster and vector images.

Table 1. Difference between Raster and Vector Images GIMP: GNU Image Manipulation Program

\begin{tabular}{|l|l|}
\hline \multicolumn{1}{|c|}{ Raster } & \multicolumn{1}{|c|}{ Vector } \\
\hline $\begin{array}{l}\text { They are composed of } \\
\text { pixels, organized to } \\
\text { form an image }\end{array}$ & $\begin{array}{l}\text { They are composed of } \\
\text { paths, guided by } \\
\text { mathematical equations } \\
\text { or formulas }\end{array}$ \\
\hline File sizes are large & File sizes are small \\
\hline $\begin{array}{l}\text { Colors can be blend } \\
\text { together and are rich }\end{array}$ & $\begin{array}{l}\text { Colors cannot be blend } \\
\text { easily without rasterizing }\end{array}$ \\
\hline $\begin{array}{l}\text { Compromised by } \\
\text { change of dimensions } \\
\text { and resolution }\end{array}$ & $\begin{array}{l}\text { Scalable to any } \\
\text { dimension and resolution }\end{array}$ \\
\hline $\begin{array}{l}\text { It is able to give } \\
\text { explicit editing }\end{array}$ & $\begin{array}{l}\text { Details are less, } \\
\text { notwithstanding provides } \\
\text { exact paths }\end{array}$ \\
\hline $\begin{array}{l}\text { Raster software } \\
\text { comprises of GIMP } \\
\text { graphics and other } \\
\text { graphics editor }\end{array}$ & $\begin{array}{l}\text { Vector } \\
\text { comprises CorelDraw, } \\
\text { Inkscape, and Illustrator }\end{array}$ \\
\hline $\begin{array}{l}\text { Graphics cost less } \\
\text {.bmp; .png, .tif, .psd, }\end{array}$ & $\begin{array}{l}\text { File types include .eps, } \\
\text { ai, .cdr, .svg, .pdf }\end{array}$ \\
\hline $\begin{array}{l}\text { Ideal for painting } \\
\text { Vector than in Raster }\end{array}$ \\
\hline Ideal for drawing \\
\hline
\end{tabular}

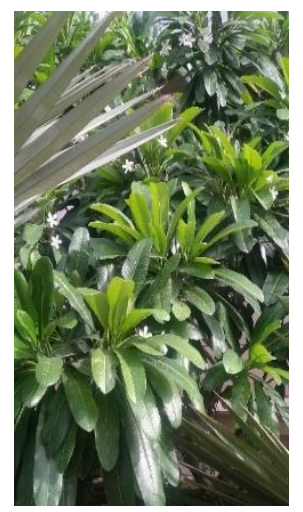
Size: $8.60 \mathrm{MB}$
Figure. 1. Raster Image

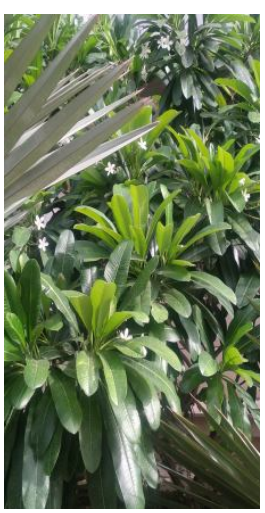

Figure. 2. Vector Image Size: $5.88 \mathrm{MB}$
As we can see, the size of the vector image is greater.

\section{ANALYSIS OF HETEROGENEOUS IMAGE FILE FORMAT}

There are numerous types of image file format, we are going to take some to analyze them below;

\subsection{TIFF (.tif, .tiff)}

TIFF stands for Tagged Image File Format. TIFF is a raster file format that uses a lossless compression, therefore it's quality or information is not lost, TIFF image quality can be restored sequel to its manipulation. TIFF is also utilized in large file sizes with high quality images. TIFF is flexible, therefore it can be lossless or lossy. Most web browsers doesn't support TIFF image format, it takes time to load the image. TIFF images uses .tif or .tiff as its extension. TIFF is extensively utilized in photography, archival record, publications and very high quality prints. A picture was taken in JPEG and converted to TIFF, we realized that the size of the image have increased from $6 \mathrm{MB}$ to $8.58 \mathrm{MB}$. Fig. 3, shows a typical example of TIFF image.

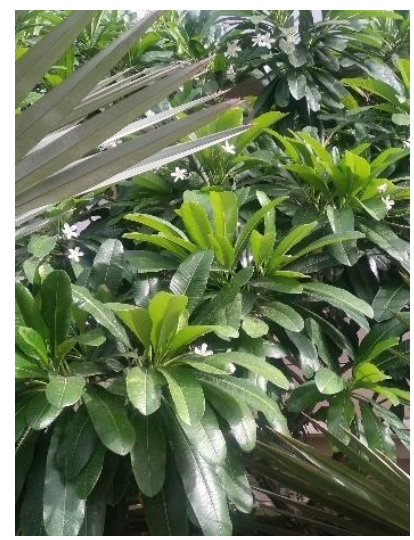

Figure. 3. TIFF Image, img.tiff Size: $8.58 \mathrm{MB}$

\subsection{Bitmap (.bmp)}

Bitmap or BMP Image File is a raster file format that was developed by Microsoft for Windows system. Bitmap file format is utilized to store bitmap images mostly on Microsoft 
Windows. Bitmap file format can store both monochrome and color image in different color depths, data compression, color profiles and alpha channels. In Bitmap file format, there is no compression or loss of information, therefore the quality of Bitmap image is extremely high but it also has a very large file size. Bitmap image is good for archival copies and high standard scans. Fig. 4, is an example of Bitmap image.

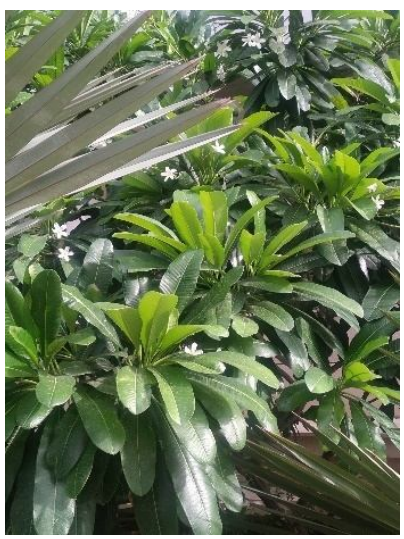

Figure. 4. BMP image,

Size: $8.6 \mathrm{MB}$

\subsection{JPEG (.jpg, .jpeg)}

The acronym "JPEG" stands for Joint Photographic Experts Group, it was developed by Joint Photographic Experts Group committee in the year 1992. JPEG is a lossy file format, which shows that the image can be compressed to get a smaller size. The degree of compression is determined by the application used. The compression of the image result to a loss of quality but in most cases it's not recognized except if the compression is greater. JPEG files are very popular on the internet and widely used format for digital cameras that supports eight-bit grayscale images and 24-bit color images (eight bits each for red, blue, and green). It is also utilized in various photographic businesses, social media and for personal use, as it is more convenient. As the file is compressed more space is created for more images to be stored on a single memory and makes it easy to transfer the file. Fig. 5, is an example of a JPEG image.

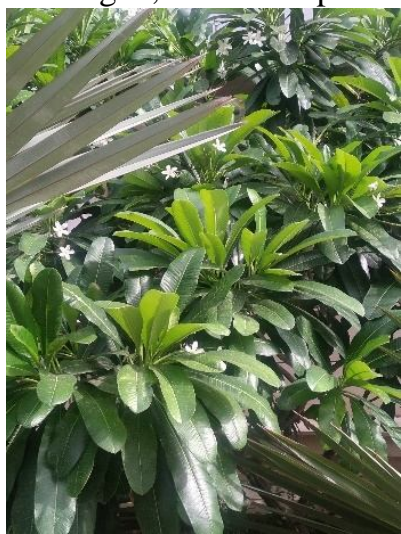

Figure. 5. JPEG image

Size: $5.90 \mathrm{MB}$

\subsection{GIF (.gif)}

GIF acronym stands for Graphics Interchange Format, it was developed by an online service provider CompuServe led by an American scientist Steve Wilhite on 15 June 1987. GIF is an image file format limited to an 8-bit palette or 256 colors. GIF is a lossless compression format. GIF is mostly used in graphics with limited colors like logos, diagrams, shapes, cartoon image, plain diagrams, web graphics, and some dithered images. GIF is commonly utilized on the World Wide Web due to its vast portability and support between operating systems and computer programs. GIFs are widely used in animation, which provides animation effects [20]. The size of GIF files are typically small and portable. GIF image format support both static and animated images. Fig. 6 is an example of GIF image.

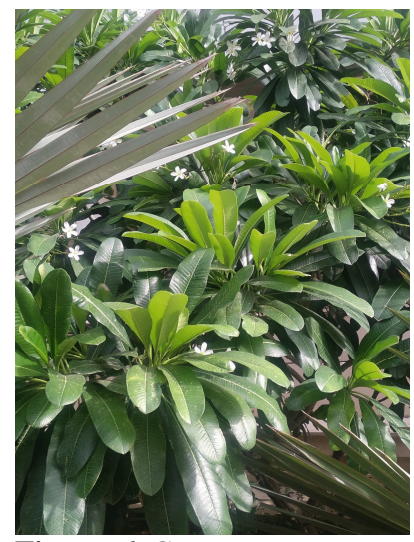

Figure. 6. GIF Image

Size: $2.32 \mathrm{MB}$

\subsection{SVG (.svg)}

The acronym SGV stands for Scalable Vector graphics. SGV is a vector file format for two-dimensional graphics format developed by World Wide Web Consortium (W3C) since 1999. SGV files are created for displaying vector graphics usually on the web. SVG represents its images using a text format based on Extensible Markup Language (XML) [21]. SVG graphics support interactivity and animation. Fig. 7, is an example of SVG image.

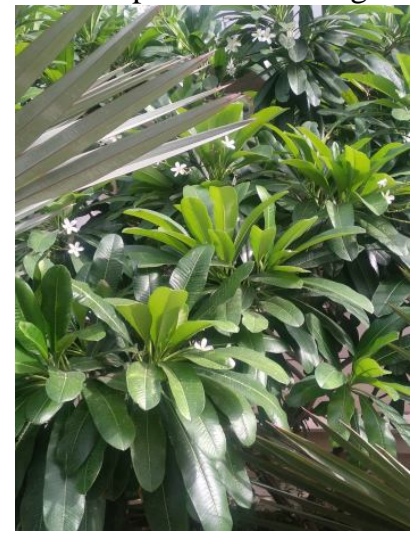

Figure. 7. SVG Image

Size: $5.88 \mathrm{MB}$ 


\subsection{PNG (.png)}

PNG acronym stands for Portable Network Graphics, is a lossless image file format designed as an open-source and a replacement of GIF format. PNG files supports eight-bit palleted images and 24-bit true color (16 million colors) as compared to GIF which supports 256 colors. PNG can store chromaticity and gamma data for enhanced color correspondence on diverse platforms. PNGs are designed to interactively work online like on web pages but not recommended for print [19]. PNG image is having a lesser size as compared to most of the image format, as we can see below the size is lesser as compared to other images. Fig. 8, is an example of PNG image.

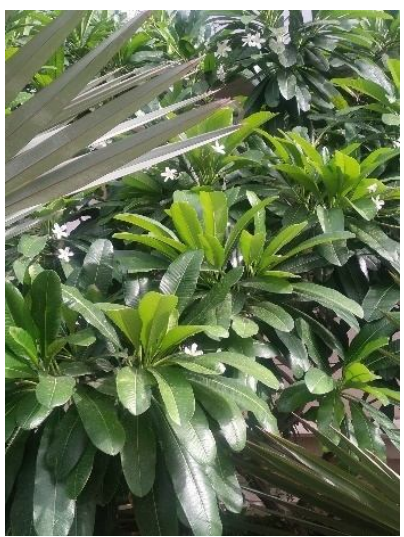

Figure. 8. PNG Image

Size: $4.35 \mathrm{MB}$

\subsection{PDF (.pdf)}

The acronym PDF stands for Portable Document Format, is an Image file format that was created by Adobe in 1993, to display documents, images, text formatting, and graphics irrespective of operating system, web browser or software. PDF is standardized as ISO 32000, it is a vector graphics but can also display in raster graphics. PDF is a sophisticated application that is not only used for documents and images but can also be used for file attachments, encryption, and metadata [23]. PDF format is usually utilized for printing purposes as it's more convenient. Fig. 9, shows a typical example of PDF image.

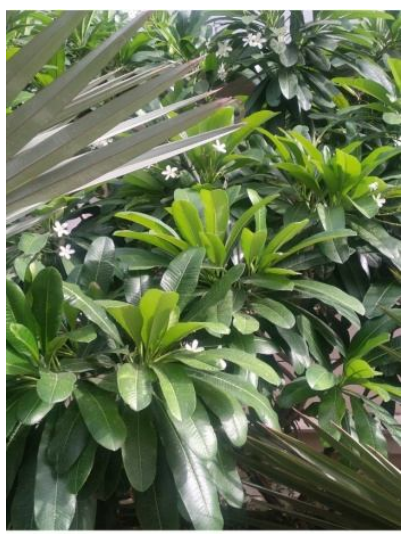

Figure. 9. PDF image

Size: $5.91 \mathrm{MB}$

\section{CONCLUSION}

While we are having different image file format available in the world today which are used for various reasons, we are able to classify this format into Raster and Vector format, we further analyzed these formats to see their properties, how they are utilized in different facet and their various applications. As digital images are saved in various Image File Formats which have different properties and features which are ideal for a particular use, we have examine the differences in image file formats, in terms of their size, graphics, flexibility, quality, and applications. We are able to conclude that Vector images are more clear than Raster images but the size of the images are large as compared to Raster, We have also realized that JPEG images are more convenient for photographic business, social media and personal use as it can be easily compressed without noticing any reduction in quality and it would save more space. PNG image is having a lesser size as compared to most of the image format therefore, it is more suitable for web pages. Choosing the right Image file format when capturing a photo is crucial. This analysis has manifested a clear picture of how the Image file formats are and how best we can utilize them for various purposes.

\section{ACKNOWLEDGEMENT}

The authors would like to acknowledge Suresh Gyan Vihar University for their support.

\section{REFERENCES}

[1] E. Abu-Taieh, A. El-Haj, A. Abu-Tayeh, A. El-Sheikh and N. Ghatasheh.Taxonomy of Image File Formats, 2013 Fourth International Conference on e-Learning "Best Practices in Management, Design and Development of eCourses: Standards of Excellence and Creativity", Bahrain, pp. 74-81, May 2013.

[2] S.Triantaphillidou, E. Allen.Digital image file formats.In the book"The Manual of Photography (Tenth Edition)"(Editors: Elizabeth Allen, Sophie Triantaphillidou), Focal Press, Chapter 17, Jan 2011, pp. 315-328.

[3] Costa, F.Z. and Dos Santos Wellington.Image file formats.In the book"Digital Document Analysis and Processing"(Editors: Carlos Alexandre Barros de Melo, Adriano Lorena Inácio de Oliveira, Wellington Pinheiro dos Santos), Nova Science, Ed. 1, June 2011, pp. 35 - 51.

[4] H. Roodaki-Lavasani, J. Lainema.Efficient burst image compression using H.265/HEVC, SPIE Electronic Imaging, San Francisco, Jan. 2014.

[5] PsPrint by deluxe, "What's the Difference Between Raster and vector?", https://www.psprint.com/resources/difference-betweenraster-vector/.

[6] R. H. Wiggins, II, H. Christian Davidson, H. Ric Harnsberger, Jason R. Lauman, Patricia A. Goede.Image File Formats: Past, Present, and Future, Radio Graphics, Vol. 21, No. 3, pp. 789-798, May 2001.

[7] D. Levine.How to Obtain Images from Picture Archiving and Communication Systems and Ready Them 
for Publication, Radiology, Vol. 257, No.3, pp. 603-608, December 2010.

[8] M. K, Image Enhancement.In the book"General Radiography: Principles and Practices"(Editors: Christopher M. Hayre, William A.S. Cox),CRC Press, Ed. 1, Chapter 3, pp. 33, July 2020.

[9] Cromey D.W.,Digital Images Are Data: And Should Be Treated as Such.In the book"Cell Imaging Techniques : Methods in Molecular Biology (Methods and Protocols)"(Editors: Taatjes D., Roth J.), Humana Press, Totowa, NJ , Vol 931, August 2012, pp. 1 -27.

[10] Fakouri R, Teimouri M.Dataset for file fragment classification of image file formats, BMC research notes, 12(1), pp. 1-3, December 2019.

[11] S. Zahra, and Mehdi Teimouri.Evaluating the Effect of Compression Settings in the Classification of Image File Formats, 2020 10th International Conference on Computer and Knowledge Engineering (ICCKE),pp. 312-316, 2020.

[12] Sakshica, K. Gupta.Various Raster and Vector Image File Formats, International Journal of Advanced Research in Computer and Communication Engineering, pp. 268-271, 2015.

[13] M.Lesk, Images of Pages.In the book"The Morgan Kaufmann Series in Multimedia Information and Systems"(Editors: Michael Lesk), Morgan Kaufmann, 2005.

[14] X. Yuan and R. Haimi-Cohen.Image Compression Based on Compressive Sensing: End-to-End Comparison With JPEG, in IEEE Transactions on Multimedia, vol. 22, no. 11, pp. 2889-2904, Nov. 2020.

[15] E. T. Eng, MykhailoKopylov, Carl J. Negro, Sarkis Dallaykan, William J. Rice, Kelsey D. Jordan, Kotaro Kelley, Bridget Carragher, Clinton S. Potter.Reducing cryoEM file storage using lossy image formats, Journal of Structural Biology, Volume 207, Issue 1, pp. 49-55,July 2019.

[16] M. M. Hannuksela, J.Lainema, V. K. MalamalVadakital.The High Efficiency Image File Format Standard [Standards in a Nutshell], in IEEE Signal Processing Magazine, vol. 32, no. 4, pp. 150-156, July 2015. [17] M. Zhang, Xiaojun Tong.A new chaotic map based image encryption schemes for several image formats, Journal of Systems and Software, Volume 98, pp. 140-154, December 2014.

[18] R.Raad Ali, and K. Malik Mohamad.RX_myKarve carving framework for reassembling complex fragmentations of JPEG images, Journal of King Saud University - Computer and Information Sciences, Volume 33, Issue 1, pp. 21-32, January 2021.

[19] Wikipedia Contributors, Wikipedia, the free encyclopedia, "PNG", available at https://en.wikipedia.org/wiki/Image_file_formats.

[20] Wikipedia Contributors, Wikipedia, the free encyclopedia, "GIF", available at https://en.wikipedia.org/wiki/GIF.

[21] Scalable Vector Graphics - Wikipedia, the free encyclopedia, "Scalable Vector Graphics", available at https://en.wikipedia.org/wiki/Scalable_Vector_Graphics.

[22] A. Almutairi.A Comparative Study on Steganography Digital Images: A Case Study of Scalable Vector Graphics (SVG) and portable Network Graphics (PNG)
Images Formats, International Journal of Advanced Computer Science and Applications, Jan 2018.

[23] Wikipedia Contributors, Wikipedia, the free encyclopedia, "PDF", available at https://en.wikipedia.org/wiki/PDF.

[24] S.Triantaphillidou, Image storage and archiving.In the book"The Manual of Photography (Tenth Edition)"(Editors: Elizabeth Allen, Sophie Triantaphillidou), Focal Press, Chapter 18, pp. 329-343, 2011.

[25] E. Allen, Image compression.In the book"The Manual of Photography (Tenth Edition)"(Editors: Elizabeth Allen, Sophie Triantaphillidou),Focal Press, Chapter 29, 2011, pp. 535-552.

[26] J. Lainema, M. M. Hannuksela, V. K. M. Vadakital and E. B. Aksu.HEVC still image coding and high efficiency image file format, 2016 IEEE International Conference on Image Processing (ICIP), pp. 71-75, 2016.

[27] Mello, Carlos \& Oliveira, Adriano \& Dos Santos, Wellington, Digital document analysis and processing, Published by Nova Science Publishers, 2012.

[28] Z. Lu, Shi-Ze Guo, Lossless Information Hiding in JPEG- and JPEG2000-Compressed Images.In the book"Lossless Information Hiding in Images"(Editors: ZheMing Lu, Shi-Ze Guo),Syngress, Chapter 6, 2017, Pp. 333402.

[29] A. Gupta et al.An Analysis of Digital Image Compression Technique in Image Processing, International Journal of Advanced Science and Technology, Vol.28, No.20, pp. 1261 -65, 2019.

[30] M. Fernando and Lu. Renfu, Basics of Image Analysis.In the book"Hyperspectral Imaging Technology in Food and Agriculture"(Editors: Mendoza Fernando and Lu. Renfu), USA, Chapter 2, January 2015, pp. $9-56$.

[31] S. Hiremath, A. Shobha Rani.A Concise Report on Image Types, Image File Format and Noise Model for Image Preprocessing, International Research Journal of Engineering and Technology (IRJET), Vol. 07, Issue 08, pp. 4991 - 4997, August 2020.

[32] Margot Note, Digital image basics. In the book "Chandos Information Professional Series" (Editors: Margot Note), Managing Image Collections, Chandos Publishing, Chapter 2, 2011, pp. 39-59.

[33] P.Akyazi and Touradj Ebrahimi.Assessment of quality of JPEG XL proposals based on subjective methodologies and objective metrics, Proc. SPIE 11137, Applications of Digital Image Processing XLII, 111370N, September 2019.

[34] The Printing CONNECTION, "Raster Images vs. Vector Graphics", available at https://www.printcnx.com/resources-and-

support/addiational-resources/raster-images-vs-vectorgraphics/.

[35] H. Cheng, Xinpeng Zhang, Jiang Yu, and Yuan Zhang.Encrypted JPEG image retrieval using block-wise feature comparison, Journal of VisualCommunication and Image Representation, Volume 40, Part A, pp. 111-117, June 2016.

[36] G. B. Daniel. "Digital Imaging", Veterinary Clinics of North America: Small Animal Practice, Volume 39, Issue 4, pp. 667-676, 2009. 
[37] H. Blitzer, Karen Stein-Ferguson, Jeffrey Huang, File Formats and Compression.In the book"Understanding Forensic Digital Imaging"(Editors: Herbert Blitzer, Karen Stein-Ferguson, Jeffrey Huang),Academic Press, Chapter 13, pp. 241-255, May 2008.

[38] M. Note, Photographic image history.In the book "Chandos Information Professional Series"(Editors: Margot Note),Managing Image Collections, Chandos Publishing, Chapter 1, 2011, pp. 15-37.

[39] K. Raman, R.Hablani, and S.Randive. Image Analysis of PLC Diagnosis System, International Journal of Advanced Trends in Computer Science and Engineering, vol. 9, no. 2, pp. 1973-1977, April 2020. 